\title{
ДВУХДИАПАЗОННАЯ Е-ОБРАЗНАЯ УНИПЛАНАРНАЯ ПЕЧАТНАЯ АНТЕННА С АСР ПИТАНИЕМ ДЛЯ СОВРЕМЕННЫХ ПРИЛОЖЕНИЙ БЕСПРОВОДНОЙ СВЯЗИ
}

\author{
П. В. НАЙДУ' \\ ${ }^{1}$ Инженерный колледж Велагапуди Рамакришна Сиддхарта, \\ Индия, Виджаявада, 520007 \\ ${ }^{2}$ Университет Симбиозис Интернешнел, \\ Индия, Пуна, 412115, Махараштра
}

\begin{abstract}
Аннотация. Предложена печатная е-образная унипланарная антенна малых размеров $(12 \times 16,5$ мм $)$ с питанием от асимметричной копланарной полосковой линии ACS (asymmetric coplanar strip), которая предназначена для двухдиапазонных систем. Многополосные рабочие характеристики реализованы путем интегрирования е-образных излучающих полосков в ACS-фидер с импедансом 50 Ом. Два одновременно работающих широких диапазона частот формируются путем использования оптимизированных излучающих полосков для многополосных систем. С целью сокращения размеров и расширения полосы пропускания, определяемой по входному импедансу, в предлагаемой конструкции антенны выбраны е-образные меандровые элементы. Предлагаемая конструкция имеет полосу пропускания (КСВН $<2$ и коэффициент отражения ниже -10 дБ), составляющую 100 МГц, в диапазоне 2,4-2,5 ГГц и 2100 МГц в диапазоне 4,0-6,1 ГГц. Разработанная многополосная антенна может использоваться для различных приложений беспроводной связи, например, системы 2,4 ГГц Bluetooth/RFID, WLAN (2,4/5,2/5,8 ГГц), WiMAX (5,5 ГГц), системы для диапазона частот служб безопасности США (4,9 ГГц), диапазона ISM (промышленный, научный и медицинский), а также систем, основанных на аккумулировании энергии высокой частоты и использовании технологии интернет вещей IоT.
\end{abstract}

Ключевые слова: двухдиапазонная антенна; асимметричная копланарная полосковая антенна; антенна с питанием ACS; асинхронная копланарная полосковая линия; несимметричная антенна; малогабаритная антенна; многополосная антенна; дешевая антенна; WLAN/WiMAX антенна

\section{1. ВВЕДЕНИЕ}

Нынешнее поколение беспроводных систем связи требует объединения многих протоколов связи в одном устройстве в пределах заданной площади или объема. При таком сценарии антенна является ключевым элементом, который определяет не только возможности устройства, но и его размеры. Чтобы справиться с этой проблемой, многие исследователи сосредоточили внимание на конструировании небольших антенн, которые одновременно работают во многих диапазонах частот.
В последнее время появились сообщения о различных конструкциях антенн, использующих разные структуры/геометрии и методы питания, например, питание от микрополосковой линии [1-3], питание от копланарного волновода CPW (coplanar waveguide) [4-8], и питание от асимметричной копланарной полосковой линии ACS (asymmetric coplanar strip) [9-19] для переносных устройств. Как показано в таблице 1 , все эти структуры отличаются сложностью и большими размерами. При этом большинство из них работают в узком диапазоне частот. 
Propag. Lett., Vol. 9, pp. 1178-1181, 2010. DOI: $10.1109 /$ LAWP.2010.2098433.

2. Flores-Leal, R.; Jardon-Aguilar, H.; Tirado-Mendez, A.; Acevo-Herrera, R. "Reduced microstrip slot multiband antenna with a U-shaped resonator for WLAN applications," Microwave Optical Technol. Lett., Vol. 54, No. 12, pp. 2684-2689, 2012. DOI: 10.1002/mop.27187.

3. Naidu, P. V. "Design, simulation of a compact triangular shaped dual-band microstrip antenna for 2.4 GHz Bluetooth/WLAN and UWB applications," Wireless Personal Commun., Vol. 95, No. 2, p. 783-794, 2017. DOI: $10.1007 / \mathrm{s} 11277-016-3798-3$.

4. Dastranj, A.; Biguesh, M. "Broadband coplanar waveguide-fed wide-slot antenna," PIER $C$, Vol. 15, pp. 89-101, 2010. DOI: 10.2528/PIERC10041706.

5. Huang, S. S.; Li, J.; Zhao, J. Z. "Compact CPW-fed tri-band antenna for WLAN/WiMAX applications," PIER C, Vol. 49, pp. 39-45, 2014. DOI: 10.2528/PIERC14030501.

6. Liu, P.; Zou, Y.; Xie, B.; Liu, X.; Sun, B. "Compact CPW-fed tri-band printed antenna with meandering split-ring slot for WLAN/WiMAX applications," IEEE Antennas Wireless Propag. Lett., Vol. 11, pp. 1242-1244, 2012. DOI: 10.1109/LAWP.2012. 2225402.

7. Liu, W.-C.; Wu, C.-M.; Chu, N.-C. "A compact CPW-fed slotted patch antenna for dual-band operation," IEEE Antennas Wireless Propag. Lett., Vol. 9, pp. 110-113, 2010. DOI: 10.1109/LAWP.2010.2044135.

8. Naidu, V. P.; Kumar, R. "Design of compact dual-band/tri-band CPW-fed monopole antennas for WLAN/WiMAX applications," Wireless Pers. Commun., Vol. 82, No. 1, p. 267-282, 2015. DOI: $10.1007 / \mathrm{s} 11277-$ 014-2207-z.

9. Ashkarali, P.; Sreenath, S.; Sujith, R.; Dinesh, R.; Krishna, D. D.; Aanandan, C. K. "A compact asymmetric coplanar strip fed dual-band antenna for DCS/WLAN applications," Microwave Optical Technol. Lett., Vol. 54, No. 4, p. 1087-1089, 2012. DOI: 10.1002/mop.26731.

10. Deepu, V.; Sujith, R.; Mridula, S.; Aanandan, C. K.; Vasudevan, K.; Mohanan, P. "ACS fed printed F-shaped uniplanar antenna for dual band WLAN applications," Microwave Optical Technol. Lett., Vol. 51, No. 8, p. 1852-1856, 2009. DOI: 10.1002/mop.24486.

11. Naidu, P. V.; Malhotra, A. "A small ACS-fed tri-band antenna employing $\mathrm{C}$ and $\mathrm{L}$ shaped radiating branches for LTE/WLAN/WiMAX/ITU wireless communication applications," Analog Integr. Circ. Sig. Process., Vol. 85, No. 3, p. 489-496, 2015. DOI: $10.1007 /$ s10470-015-0637-5.

12. Deepu, V.; Raj, R. K.; Manoj, Joseph; Suma, M. N.; Mohanan, P. "Compact asymmetric coplanar strip fed monopole antenna for multiband applications," IEEE Trans. Antennas Propag., Vol. 55, No. 8, p. 2351-2357, Aug. 2007. DOI: 10.1109/TAP.2007.901847.

13. Naidu, P. V. „Printed V-shape ACS-fed compact dual band antenna for bluetooth, LTE and WLAN/WiMAX applications," Microsyst. Technol., Vol. 
23, No. 4, p. 1005-1015, 2017. DOI: $10.1007 / \mathrm{s} 00542-016-$ 2939-7.

14. Li, Y.; Li, W.; Mittra, R. „Miniaturization of ACS-fed dual-band antenna with loaded capacitance terminations for WLAN applications," IEICE Electron. Expr., Vol. 10, No. 15, p. 20130455-20130455, 2013. DOI: $10.1587 /$ elex.10.20130455.

15. Naidu, P. V.; Kumar, R. "A very small asymmetric coplanar strip fed multi-band antenna for wireless communication applications," Microsyst. Technol., Vol. 22, No. 9, p. 2193-2200, 2015. DOI: $10.1007 / \mathrm{s} 00542-0$ 15-2613-5.

16. Chen, L.; Luo, Y.-L.; Zhang, Y. "Compact tri-band planar monopole antenna with ACS-fed structure," PIER Lett., Vol. 49, p. 45-51, 2014. DOI: 10.2528/ PIERL14072207.

Поступила в редакцию 16.12.2016
17. Ansal, K. A.; Shanmuganatham, T. "ACS-fed wide band antenna with L-shaped ground plane for 5.5 GHz WLAN application," PIER Lett., Vol. 49, p. 59-64. DOI: $10.2528 /$ PIERL14053106.

18. Naidu, P. V.; Malhotra, A.; Kumar, R. "A compact ACS-fed dual-band monopole antenna for LTE, WLAN/WiMAX and public safety applications," Microsyst. Technol., Vol. 22, No. 5, p. 1021-1028, 2016. DOI: $10.1007 / \mathrm{s} 00542-015-2562-\mathrm{z}$.

19. Choukiker, Y. K.; Behera, S. K. "ACS fed Koch fractal antenna for wide-band applications," Int. J. Signal Imaging Systems Engineering, Vol. 6, No. 1, p. 9-15, 2013. DOI: $10.1504 /$ ijsise. 2013.051500 .

20. Simons, R. N. Coplanar Waveguide Circuits, Components, and Systems. John Wiley \& Sons, 2001. DOI: $10.1002 / 0471224758$.

После переработки 26.09.2017 